\title{
Cloud Point Curve and Critical Point of Multicomponent Polymer/Single Solvent System
}

\author{
Kenji KAMIDE, Shigenobu MATSUdA, Toshiaki DobASHI, ${ }^{*, \dagger}$ \\ and Motozo KANEKO* \\ Textile Research Laboratory, Asahi Chemical Industry Co., Ltd., \\ 11-7, Hacchonawate, Takatsuki, Osaka 569, Japan \\ * Department of Polymer Science, Hokkaido University, \\ Sapporo 060, Japan
}

(Received April 11, 1984)

\begin{abstract}
An attempt was made to establish a theoretical method for calculating the cloud point curve (CPC) and critical point of solutions of polydisperse polymers in a single solvent (i.e., quasi-binary system) on the basis of the polydispersity of the polymer and concentration- and molecular weight-dependences of the polymer-solvent thermodynamic interaction parameter $\chi$. Expressions giving the cloud point curve were derived and a computer simulation technique, based on the theory, was developed. The effects of the average molecular weight, polydispersity of polymer and concentration dependence of $\chi$-parameter on CPC, threshold cloud point and critical point were clarified. In order to represent accurately the CPC for the entire concentration range from the molecular characteristics of the original polymer and operating conditions, such as polymer-volume fraction and $\chi$-parameter, the concentration dependence parameters $p_{1}$ and $p_{2}$, in the relation $\chi=\chi_{0}\left(1+p_{1} v_{\mathrm{p}}+p_{2} v_{\mathrm{p}}{ }^{2}\right)\left(\chi_{0}\right.$; concentration-independent parameter, $v_{\mathrm{p}}$, polymer volume fraction) should be adequately taken into account. Very delicate changes in $p_{1}$ and $p_{2}$ cause significant variation in CPC and none of the literature data on $p_{1}$ and $p_{2}$ for polystyrene/ cyclohexane adequately represent the experimental CPC. From an actual CPC experiment, $p_{1}=$ 0.643 and $p_{2}=0.200$ were evaluated. The effective role of the molecular weight dependence of $\chi$ in $\mathrm{CPC}$ was shown in the lower $v_{\mathrm{p}}$ region.

KEY WORDS Cloud Point Curve / Polydispersity / Concentration Dependence / Molecular Weight Dependence / $\chi$ Parameter / Polystyrene / Computer Simulation / Threshold Cloud Point / Critical Point / QuasiBinary System /
\end{abstract}

The cloud point curve (CPC) of polymer/ single solvent system is experimentally known to be highly unsymmetrical with respect to polymer concentration, regardless of the polymolecularity of the polymer. ${ }^{1,2}$ This high degree of dissymmetry in CPC for monodisperse polymer/solvent system can be explained theoretically in terms of the large molar volume ratio $\mathrm{X}$ of the polymer to the solvent. For the strictly monodisperse polymer solution (if available) the coexistence curve (CC) should be in excellent agreement with CPC. As early as 1952, Shultz and Flory ${ }^{3}$ determined experimentally the CPC for polydisperse polyisobutyrene/diisobutyl ketone and polystyrene/cyclohexane systems, comparing CC instead of CPC for these systems, using the Flory-Huggins type thermodynamic theory for polymer solutions and assuming a monodisperse polymer/single solvent system and a constant thermodynamic interaction parameter $\chi$ over a wide range of concentration (see Figures 2 and 3 of ref 3). They noticed that the shapes of the calculated CPC agreed only

\footnotetext{
${ }^{\dagger}$ Present address: Department of Polymer Technology, Gunma University, Kiryu, Gunma, Japan.
} 
quantitatively with those observed and the latter was markedly broader and the observed critical points occurred at higher polymer concentrations than those predicted. The significant divergence of the observed CPC from the simple thermodynamic theory may be due to (1) the polymolecularity of the polymers and (2) the concentration dependence of the thermodynamic interaction parameter $\chi$, because an important role of $\chi$-parameter in the phase equilibrium of polymer solutions as shown by Kamide et al. ${ }^{4-12}$ Unfortunately, the effect of the polymolecularity of the polymer and concentration dependence of the $\chi$-parameter on CPC and CC could not be correctly estimated in 1952 due to the lack of theoretical background of phase equilibrium of multicomponent polymer solutions. Shultz and Flory determined also experimentally the CPC for a mixture of two polystyrene fractions (the relative molar volume ratio of the polymer to the solvent $\mathrm{X}=768$ and $1.095 \times 10^{4}$ ) dissolved in cyclohexane, pointing out that the CPC of this quasi-ternary system is depressed in a higher concentration range than the precipitation threshold point.

Rehage et al..$^{13}$ estimated the cloud point as the cross point of a CC, obtained for a multicomponent polystyrene-cyclohexane system with a given initial polymer volume fraction $v_{\mathrm{p}}^{0}$, and the $v_{\mathrm{p}}^{0}$ axis. Kuwahara et al. ${ }^{14}$ measured directly the CPC of five polystyrenes dissolved in cyclohexane near their critical points, showing experimentally that the polymer volume fractions at the precipitation threshold point $\left(v_{\mathrm{p}, \mathrm{tcp}}\right)$ are significantly smaller than those at the critical point $\left(v_{\mathrm{p}}^{\mathrm{c}}\right)$ even for a solution of "monodisperse" polystyrene characterized by the ratio of the weight- to the number-average molecular weight $M_{w} / M_{n}<1.03$. Koningsveld and Staverman ${ }^{15-17}$ applied Rehage et al.'s procedure for estimating the cloud point to their calculations using log-normal, bimodal, and exponential type molecular weight distributions for the original polymer.

At the present, spinodal and coexistence curves can be calculated for multicomponent polymer/single solvent systems, ${ }^{4,9,15,17^{-21}}$ But, there is no example of a direct theoretical calculation of CPC. The indirect method originally proposed by Rehage et al. is strongly limited and applicable to an extremely limited range. Rehage et al.'s procedure, as expected, very tedious and time consuming, because a single coexistence curve yields only a single point in a CPC. Systematic study on CPC has not been pursued very much.

This article attempts to establish a theoretical method for directly evaluating CPC without reliance on a coexistence curve and carry out the computer experiment to clarify the effects of the molecular characteristics of the original polymer and of concentration- and molecular weight-dependence of the $\chi$-parameter on CPC and compare the observed CPC for a multicomponent polystyrenecyclohexane system with that theoretically determined.

The critical solution point of the polydisperse polymer solution in a single solvent was first studied by Stockmayer who did not consider the concentration dependence of the $\chi$ parameter. The most rigorous expressions for critical points were derived by Koningsveld and Staverman, ${ }^{22}$ who considered the concentration dependence of the $\chi$-parameter along with the polymolecularity of the polymer. Koningsveld et al. ${ }^{23,34}$ applied their theory to estimate the concentration dependence of the $\chi$-parameter. Unfortunately, no systematic treatment has been given for critical solution points. In this paper, we also extend the theory to the case in which the polymolecularity of a polymer and the concentration- and molecular weight-dependence of $\chi$-parameter exist for a quasibinary mixture and show the effects of these factors on the critical points in some detail.

\section{THEORETICAL BACKGROUND}

Since 1968, computer experiments based on 
the rigorous theory have been energetically carried out on multicomponent polymer/single solvent systems by Kamide and his coworkers $^{4,9,11,20,21}$ and Koningsveld et al. ${ }^{15-19}$ Kamide et al. ${ }^{11}$ expressed, in a purely phenomenological sense, the thermodynamic interaction parameter $\chi_{i}$ of the $i$-th polymer component in the form

$$
\begin{array}{r}
\chi_{i}=\chi_{00}\left(1+k^{\prime} / X_{i}\right)\left(1+p v_{\mathrm{p}}\right) \\
(i=1,2, \cdots, m)
\end{array}
$$

where $\chi_{00}$ is a parameter independent of the polymer volume fraction $v_{\mathrm{p}}$ and the molar volume ratio of the $i$-th component to the solvent $X . k^{\prime}$ and $p$ are molecular weight- and the concentration-independent parameters. $\chi_{00}$ and $k^{\prime}$ are functions of temperature and probably of pressure. $m$ is the total number of components. They confirmed experimentally that eq 1 is accurate enough to represent the two-phase equilibrium of a multicomponent polymer-single solvent system and disclose the effects of the $k^{\prime}$ and $p$ parameters on the characteristics of coexistence curve in detail. In other words, although a higher term containing $v_{\mathrm{p}}{ }^{2}$ can be observed in the $\chi$-parameter by membrane osmometry and vapor pressure measurements, the term does not significantly contribute to the phase separation characteristics including coexistence curve, within experimental certainty.

In this article, we express $\chi_{i}$ in the generalized form of eq 1 ,

$$
\begin{aligned}
\chi_{i}=\chi_{00}\left(1+k^{\prime} / X_{i}\right)\left(1+\sum_{j=1}^{n} p_{j} v_{\mathrm{p}}^{j}\right) & \\
& (i=1, \cdots, m)
\end{aligned}
$$

Assuming that (1) the enthalpy change results from contact of solvent and solute and not from contact of solutes with different $X_{i}$ and (2) the Bragg-Williams approximation is applicable to the solution, we can write the heat of dilution $\Delta H$ of the solution consisting multicomponent polymers (total number of the component $m$ ) and a single solvent by

$$
\Delta H=\tilde{R} T\left\{\chi_{00}\left(1+k^{\prime} / X_{n}\right)\left(1+\sum_{j=1}^{n} p_{j} v_{\mathrm{p}}^{j}\right)\right\} v_{0} v_{\mathrm{p}}
$$

whre $\tilde{R}$ is the gas constant, $T$, temperature $(K)$, $X_{n}$, the number average $X$, and $v_{0}$, the solvent volume fraction $\left(=1-v_{\mathrm{p}}\right)$. From eq 3 the partial molar heat of dilution $\Delta H_{0}$ can be directly derived.

Using eq 3, the chemical potential of the solvent $\Delta \mu_{0}$ can be rewritten as

$$
\begin{aligned}
\Delta \mu_{0}= & \tilde{R} T\left\{\ln \left(1-v_{\mathrm{p}}\right)+\left(1-\frac{1}{X_{n}}\right) v_{\mathrm{p}}\right. \\
& \left.+\chi_{00}\left(1+\frac{k^{\prime}}{X_{n}}\right)\left(1+\sum_{j=1}^{n} p_{j} v_{\mathrm{p}}^{j}\right) v_{\mathrm{p}}^{2}\right\}
\end{aligned}
$$

The chemical potential of $X_{i}$-mer $\Delta \mu_{i}$ is derived from eq 4 when these potentials satisfy the Gibbs-Duhem equation. The results are

$$
\begin{aligned}
\Delta \mu_{i}= & \tilde{R} T\left[\ln v_{i}-\left(X_{i}-1\right)+X_{i}\left(1-\frac{1}{X_{n}}\right) v_{\mathrm{p}}\right. \\
& +X_{i}\left(1-v_{\mathrm{p}}\right)^{2} \chi_{00}\left[\left(1+\frac{k^{\prime}}{X_{n}}\right)\right. \\
& \times\left\{1+\sum_{j=1}^{n} \frac{p_{j}}{j+1}\left(\sum_{q=0}^{j}(q+1) v_{\mathrm{p}}^{q}\right)\right\} \\
& +k^{\prime}\left(\frac{1}{X_{i}}-\frac{1}{X_{n}}\right) \\
& \left.\left.\times\left\{\frac{1}{1-v_{\mathrm{p}}}+\sum_{j=1}^{n} \frac{p_{j}}{j+1}\left(\sum_{q=0}^{j} \frac{v_{\mathrm{p}}^{q}}{1-v_{\mathrm{p}}}\right)\right\}\right]\right]
\end{aligned}
$$

A detailed derivation of eq 5 is given in Appendix A. Note that the applicability of eq 5 is not ristricted only to the lattice model used originally in the Flory-Huggins theory.

The well-known Gibbs's conditions for the phase equilibrium of a quasi-binary solution at constant temperature and pressure are

$$
\begin{aligned}
& \Delta \mu_{0(1)}=\Delta \mu_{0(2)} \\
& \Delta \mu_{i(1)}=\Delta \mu_{i(2)} \quad(i=1, \cdots, m)
\end{aligned}
$$

Here, the suffixes (1) and (2) denote the polymer-lean and the rich phases, respectively. 
Combining eq $4-7$ yields

$$
\begin{aligned}
\chi_{00}= & \left\{\ln \frac{1-v_{\mathrm{p}(1)}}{1-v_{\mathrm{p}(2)}}+\left(v_{\mathrm{p}(1)}-v_{\mathrm{p}(2)}\right)\right. \\
& \left.-\left(\frac{v_{\mathrm{p}(1)}}{X_{n(1)}}-\frac{v_{\mathrm{p}(2)}}{X_{n(2)}}\right)\right\} /\left\{\left(v_{\mathrm{p}(2)}^{2}-v_{\mathrm{p}(1)}^{2}\right)\right. \\
& +k^{\prime}\left(\frac{v_{\mathrm{p}(2)}^{2}}{X_{n(2)}}-\frac{v_{\mathrm{p}(1)}^{2}}{X_{n(2)}}\right) \\
& \left.+\sum_{j=1}^{n} p_{j}\left(v_{\mathrm{p}(2)}^{j+2}-v_{\mathrm{p}(1)}^{j+2}\right)+k^{\prime}\left(\frac{v_{\mathrm{p}(2)}^{j+2}}{X_{n(2)}}-\frac{v_{\mathrm{p}(1)}^{j+2}}{X_{n(1)}}\right)\right\}
\end{aligned}
$$

and

$$
\sigma_{i} \equiv \frac{1}{X_{i}} \ln \frac{v_{i(2)}}{v_{i(1)}}=\sigma_{0}+\sigma_{01} / X_{i}
$$

with

$$
\begin{aligned}
\sigma_{0} & =\left(v_{\mathrm{p}(1)}-v_{\mathrm{p}(2)}\right)-\left(\frac{v_{\mathrm{p}(1)}}{X_{n(1)}}-\frac{v_{\mathrm{p}(2)}}{X_{n(2)}}\right) \\
& +\chi_{00}\left[2\left(v_{\mathrm{p}(1)}-v_{\mathrm{p}(2)}\right)-\left(v_{\mathrm{p}(1)}^{2}-v_{\mathrm{p}(2)}^{2}\right)\right. \\
& \left.+\sum_{j=1}^{n} p_{j}\left\{\frac{j+2}{j+1}\left(v_{\mathrm{p}(1)}^{j+1}-v_{\mathrm{p}(2)}^{j+1}\right)-\left(v_{\mathrm{p}(1)}^{j+2}-v_{\mathrm{p}(2)}^{j+2}\right)\right\}\right] \\
& -\chi_{00} k^{\prime}\left[\left(\frac{v_{\mathrm{p}(1)}}{X_{n(1)}}-\frac{v_{\mathrm{p}(2)}}{X_{n(2)}}\right)-\left(\frac{v_{\mathrm{p}(1)}^{2}}{X_{n(1)}}-\frac{v_{\mathrm{p}(2)}^{2}}{X_{n(2)}}\right)\right. \\
& \left.-\sum_{j=1}^{n} p_{j}\left\{\left(\frac{v_{\mathrm{p}(1)}^{j+1}}{X_{n(1)}}-\frac{v_{\mathrm{p}(2)}^{j+1}}{X_{n(2)}}\right)-\left(\frac{v_{\mathrm{p}(1)}^{j+2}}{X_{n(1)}}-\frac{v_{\mathrm{p}(2)}^{j+2}}{X_{n(2)}}\right)\right\}\right]
\end{aligned}
$$

and

$$
\sigma_{01}=k^{\prime}\left\{\left(v_{\mathrm{p}(1)}-v_{\mathrm{p}(2)}\right)+\sum_{j=1}^{n} \frac{p_{j}}{j+1}\left(v_{\mathrm{p}(1)}^{j+2}-v_{\mathrm{p}(2)}^{j+2}\right)\right\}
$$

When $k^{\prime}=0$, eq 2 reduces to

$$
\chi=\chi_{0}\left(1+\sum_{j=1}^{n} p_{j} v_{\mathrm{p}}^{j}\right)
$$

The polymer-solvent interaction parameter $g$, introduced in Koningsveld et al.'s theory, ${ }^{22}$ can be expressed as a power function of $v_{\mathrm{p}}$,

$$
g=\sum_{i=0}^{n} g_{i} v_{\mathrm{p}}^{i}
$$

where $g_{i}$ is a function of temperature and independent of concentration.

If both $\chi$ and $g$ have only concentration dependence and no molecular weight dependence, $\chi$ is related to $g$ through

$$
\chi=g-\left(1-v_{\mathrm{p}}\right) \frac{\partial g}{\partial v_{\mathrm{p}}}
$$

From eq 11-13, we obtain

$$
\begin{aligned}
& \chi_{0}=g_{0}-g_{1} \\
& p_{i}=(i+1)\left(g_{i}-g_{i+1}\right) /\left(g_{0}-g_{1}\right) \\
& \qquad(i=1, \cdots, n-1) \\
& p_{n}=(n+1) g_{n} /\left(g_{0}-g_{1}\right)
\end{aligned}
$$

Koningsveld and Kleintjens ${ }^{23}$ expressed $g$ in the closed form of $v_{\mathrm{p}}$, given by

$$
g=\alpha+\beta /\left(1-\gamma v_{\mathrm{p}}\right)
$$

where $\alpha, \beta$, and $\gamma$ are parameters. In the range $0<\gamma<1$, the relation $0<\gamma v_{\mathrm{p}}<1$ is valid and the second term in the right-hand side of eq 17 can be expanded in infinite series so that eq 17 may be rewritten as,

$$
\begin{aligned}
g & =\alpha+\beta+\sum_{i=1}^{\infty} \beta\left(\gamma v_{\mathrm{p}}\right)^{i} \\
& \simeq \alpha+\beta+\sum_{i=1}^{n} \beta\left(\gamma v_{\mathrm{p}}\right)^{i}
\end{aligned}
$$

By putting $\alpha+\beta=g_{0}$ and $\beta \gamma^{i}=g_{i} \quad(i=$ $1, \cdots, n)$ eq 18 is reduced to eq 12. Parameters $\chi_{0}, p_{i}$, and $p_{n}$ can be expressed in terms of $\alpha, \beta$, and $\gamma$ as follows.

$$
\begin{aligned}
& \chi_{0}=\alpha+\beta(1-\gamma) \\
& p_{i}=(i+1) \beta \gamma^{i}(1-\gamma) /\{\alpha+\beta(1-\gamma)\} \\
& \qquad(i=1, \cdots, n-1) \\
& p_{n}=(n+1) \beta \gamma^{n} /\{\alpha+\beta(1-\gamma)\}
\end{aligned}
$$

The spinodal is determined by 


$$
\left|\Delta G^{\prime}\right| \equiv\left|\begin{array}{cccc}
\overline{\Delta G}_{11}^{\prime} & \overline{\Delta G}_{12}^{\prime} & \cdots & \overline{\Delta G}_{1 m}^{\prime} \\
\overline{\Delta G}_{21}^{\prime} & \overline{\Delta G}_{22}^{\prime} & \cdots & \overline{\Delta G}_{2 m}^{\prime} \\
\vdots & & & \\
\overline{\Delta G}_{m 1}^{\prime} & \overline{\Delta G}_{m 2}^{\prime} & \cdots & \overline{\Delta G}_{m m}^{\prime}
\end{array}\right|=0
$$

and this can be combined with

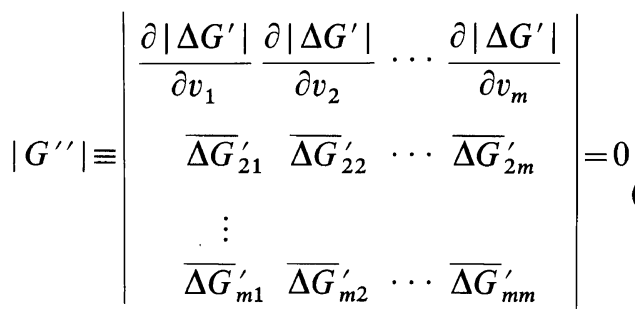

to obtain the critical solution point for multicomponent polymer/single solvent systems. ${ }^{18}$ In eq 22 and $23, \Delta G^{\prime}$ is the Gibbs free energy change of mixing per unit volume, given by

$$
\Delta G^{\prime}=v_{0}\left(\frac{\Delta \mu_{0}}{V_{0}}\right)+\sum_{i=1}^{m} v_{i}\left(\frac{\Delta \mu_{i}}{X_{i} V_{0}}\right)
$$

and $\overline{\Delta G}_{i j}^{\prime}$ is defined by eq 25

$$
\overline{\Delta G_{i j}^{\prime}}=\left(\frac{\partial^{2} \Delta G^{\prime}}{\partial v_{i} \partial v_{j}}\right)_{T, p} \quad(i, j=1, \cdots, m)
$$

where $V_{0}$ is the molar volume of solvent, and $v_{i}$, the volume fraction of $X_{i}$-mer. The differentiation is carried out at constant temperature and pressure.

We attempted to solve eq 22 and 23 in a case where $\chi$ depends on molecular weight as well as concentration, as expressed by eq 1 .

Substituting eq 4 and 5 into eq 24, we obtain

$$
\begin{aligned}
\Delta G^{\prime}= & \frac{\tilde{R} T}{V_{0}}\left[\left(1-v_{\mathrm{p}}\right) \ln \left(1-v_{\mathrm{p}}\right)+\sum_{i=1}^{m} \frac{v_{i} \ln v_{i}}{X_{i}}\right. \\
& +\chi_{00}\left\{1+\frac{k^{\prime}}{X_{n}}\left(1+X_{n}-X_{w}\right)\right\} \\
& \left.\times\left\{1+\sum_{j=1}^{n} \frac{p_{j}}{j+1}\left(\sum_{q=0}^{j} v_{\mathrm{p}}^{q}\right)\right\} v_{\mathrm{p}}\left(1-v_{\mathrm{p}}\right)\right]
\end{aligned}
$$

Here, $X_{w}$ is the weight-average $X_{i}$.

Considering eq 25 and combining eq 26 with eq 22 and 23 , we finally obtain eq 27 and 28 . (Appendix B) to give the composition of a critical solution point.

$$
\begin{gathered}
\frac{1}{X_{w} v_{\mathrm{p}}}+\frac{1}{1-v_{\mathrm{p}}}-\chi_{00}\left\{1+k^{\prime}\left(1+\frac{1}{X_{n}}-\frac{X_{w}}{X_{n}}\right)\right\} \\
\times\left\{2+\sum_{j=1}^{n} p_{j}(j+2) v_{\mathrm{p}}^{j}\right\}=0 \\
\frac{1}{\left(1-v_{\mathrm{p}}\right)^{2}}-\frac{X_{z}}{\left(X_{w} v_{\mathrm{p}}\right)^{2}}-\chi_{00}\left\{1+k^{\prime}\left(1+\frac{1}{X_{n}}-\frac{X_{w}}{X_{n}}\right)\right\} \\
\times\left\{\sum_{j=1}^{n} p_{j} j(j+2) v_{\mathrm{p}}^{j-1}\right\}=0
\end{gathered}
$$

where $X_{z}$ is the $z$-average $X$. Both $v_{\mathrm{p}}$ and $\chi_{00}$ at critical points, are referred to as $v_{\mathrm{p}}^{\mathrm{c}}$ and $\chi_{00}^{\mathrm{c}}$, and can be calculated by application of eq 27 and 28 from $X_{w}, X_{z}$, of the original polymer $\left(X_{w}^{0}\right.$ and $\left.X_{z}^{0}\right) k^{\prime}$ and $p_{j}(j=1, \cdots, n)$.

The temperature dependence of $k^{\prime}$ and $\chi_{00}$ can be empirically expressed as

$$
k^{\prime}=k_{0}(1-\theta / T)
$$

and

$$
\chi_{00}=a+b / T
$$

where $k_{0}, a$, and $b$ are constants independent of temperature and $\theta$, the Flory temperature. ${ }^{12}$ The concentration and temperature dependence of the $\chi$-parameter are thus given by

$$
\begin{aligned}
\chi= & \left(a^{\prime}+b^{\prime} / T+c^{\prime} / T^{2}\right) \\
& \times\left(1+p_{1} v_{\mathrm{p}}+p_{2} v_{\mathrm{p}}{ }^{2}\right)
\end{aligned}
$$

with

$$
\begin{aligned}
& a^{\prime}=a\left(1+k_{0} / X_{n}\right) \\
& b^{\prime}=b+\left(k_{0} / X_{n}\right)(b-a \theta) \\
& c^{\prime}=-\left(k_{0} b \theta / X_{n}\right)
\end{aligned}
$$

Equation 31 is readily derived by comparing eq 11,29 , and 30 . When $k_{0}=0, a$ reduces to $a$, $b^{\prime}$ to $b$ and $c^{\prime}$ to zero. 


\section{COMPUTER SIMULATION OF CPC}

The volume fractions of the $X_{i}$-mer in both phases, $v_{(1)}\left(X_{i}\right)$ and $v_{(2)}\left(X_{i}\right)$, are given by

$$
\begin{gathered}
v_{(1)}\left(X_{i}\right)=\frac{R+1}{R+\exp \left(\sigma_{i} X_{i}\right)} v_{0}\left(X_{i}\right) \\
v_{(2)}\left(X_{i}\right)=\frac{(R+1) \exp \left(\sigma_{i} X_{i}\right)}{R+\exp \left(\sigma_{i} X_{i}\right)} v_{0}\left(X_{i}\right)
\end{gathered}
$$

where $R$ is the ratio of the volume of the polymer-lean phase $V_{(1)}$ to that of the polymerrich phase $V_{(2)}$ and $v_{0}\left(X_{i}\right)$ is the volume fraction of $X_{i}$-mer in the starting solution. The $v_{(1)}\left(X_{i}\right)$ and $v_{(2)}\left(X_{i}\right)$ at the cloud point, (referred to as $\left.v_{(1)}{ }^{c p}\left(X_{i}\right), v_{(2)}{ }^{c p}\left(X_{i}\right)\right)$, are given from eq 33 and 34 as limiting cases of $R \rightarrow \infty$ when the polymer volume fraction of the starting solution $v_{\mathrm{p}}^{0}$ is lower than $v_{\mathrm{p}}^{\mathrm{c}}\left(\right.$ i.e., $\left.v_{\mathrm{p}}^{0}<v_{\mathrm{p}}^{\mathrm{c}}\right)$ and $R \rightarrow 0$ for $v_{\mathrm{p}}^{0}>v_{\mathrm{p}}^{\mathrm{c}}$ in the form:

$$
\begin{array}{ll}
\text { (1) } v_{(1)}^{c p}\left(X_{i}\right)=v_{0}\left(X_{i}\right) \\
\\
v_{(2)}^{c p}\left(X_{i}\right)=v_{0}\left(X_{i}\right) \exp \left(\sigma_{i} X_{i}\right) \\
\text { for } v_{\mathrm{p}}^{0}<v_{\mathrm{p}}^{\mathrm{c}} \quad(i=1, \cdots, m) \quad(35) \\
\text { (2) } v_{(1)}^{c p}\left(X_{i}\right)=v_{0}\left(X_{i}\right) \exp \left(-\sigma_{i} X_{i}\right) \\
\\
v_{(2)}^{c p}\left(X_{i}\right)=v_{0}\left(X_{i}\right) \\
\text { for } v_{\mathrm{p}}^{0}>v_{\mathrm{p}}^{\mathrm{c}} \quad(i=1, \cdots, m)
\end{array}
$$

Thus, on the basis of eq $8-10,35$, and 36 , $\chi_{00}$ at cloud point $\left(\chi_{00}^{c p}\right), \sigma, v_{(1)}\left(X_{i}\right)$ and $v_{(2)}\left(X_{i}\right)$, $v_{\mathrm{p}(1)}$ and $v_{\mathrm{p}(2)}$ can be directly calculated for a given $v_{\mathrm{p}}^{0}$ solution. A brief flow chart presentation of the computer simulation is shown in Figure 1. The prerequisites for this simulation are,

(a) molecular weight distribution and accordingly the weight- and the number-average $X$ of the original polymer $\left(X_{w}^{0}\right.$ and $\left.X_{w}^{0} / X_{n}^{0}\right)$

(b) polymer volume fraction of the starting solution $v_{\mathrm{p}}^{0}$

(c) parameters $k_{0}$ and $\theta$ in eq 29

(d) concentration dependence parameters $p_{j}(j=1, \cdots, n)$ in eq 1 .
We can obtain $v_{\mathrm{p}}^{\mathrm{c}}$ and $\chi_{00}^{\mathrm{c}}$ by resolving the simultaneous equations (eq 27 and 28) using Newton's procedure. In this calculation, as the initial value of $v_{\mathrm{p}}^{\mathrm{c}}$, the critical value given by the Stockmayer equation, ${ }^{25}$

$$
v_{\mathrm{p}}^{\mathrm{c}}=\frac{1}{1+X_{w}\left(X_{z}\right)^{-1 / 2}}
$$

was taken.

\section{Computer Experiment 1}

To clarify the effects of $X_{w}^{0}, X_{w}^{0} / X_{n}^{0}, p_{1}$, and $p_{2}$ on CPC, a series of computer experiments was carried out. The original polymers were assumed to have the Schulz-Zimm (SZ) type molecular weight distribution with $X_{w}^{0}$ in the range of 300 to 5000 and with $X_{w}^{0} / X_{n}^{0}$ ranging from 1.03 to 4 , having polymer components of 1500 to 6000 , differing in $X$. The following parameters were employed for the claculation: $v_{\mathrm{p}}^{0}=0.0005-0.15, k^{\prime}=0, a=0.0481$ and $b=$ $137.2, p_{1}=0-0.8$, and $p_{2}=0-0.6$.

\section{Computer Experiment 2}

For a comparison with the actual experiment on a polystyrene/cyclohexane system, $\mathrm{CPC}$ was calculated under the same conditions as those in the actual experiment. The polymer had the Schulz-Zimm type distribution with $X_{w}^{0}=2117, X_{w}^{0} / X^{0}=2.8$ for cyclohexane.

\section{DETERMINATION OF PARAMETERS

$$
a, b, \text { AND } p_{j}
$$

Parameters $a, b$, and $p_{j}(j=1, \cdots, n)$ can be unambiguously determined when the cloud point temperarure $T_{\mathrm{cp}, \mathrm{e}}$, experimentally determined, coincides with that calculated by computer simulation, $T_{\mathrm{cp}, \mathrm{c}}$, for any solution. In this study the following procedure was used to evaluate these parameters:

(1) Determine the relations between $\rho_{\mathrm{p}}\left(\rho_{\mathrm{p}}\right.$ is the weight fraction of the polymer partitioned into the polymer-rich phase to the polymer dissolved in the initial solution). and $T$ from the two-phase equilibrium experi- 


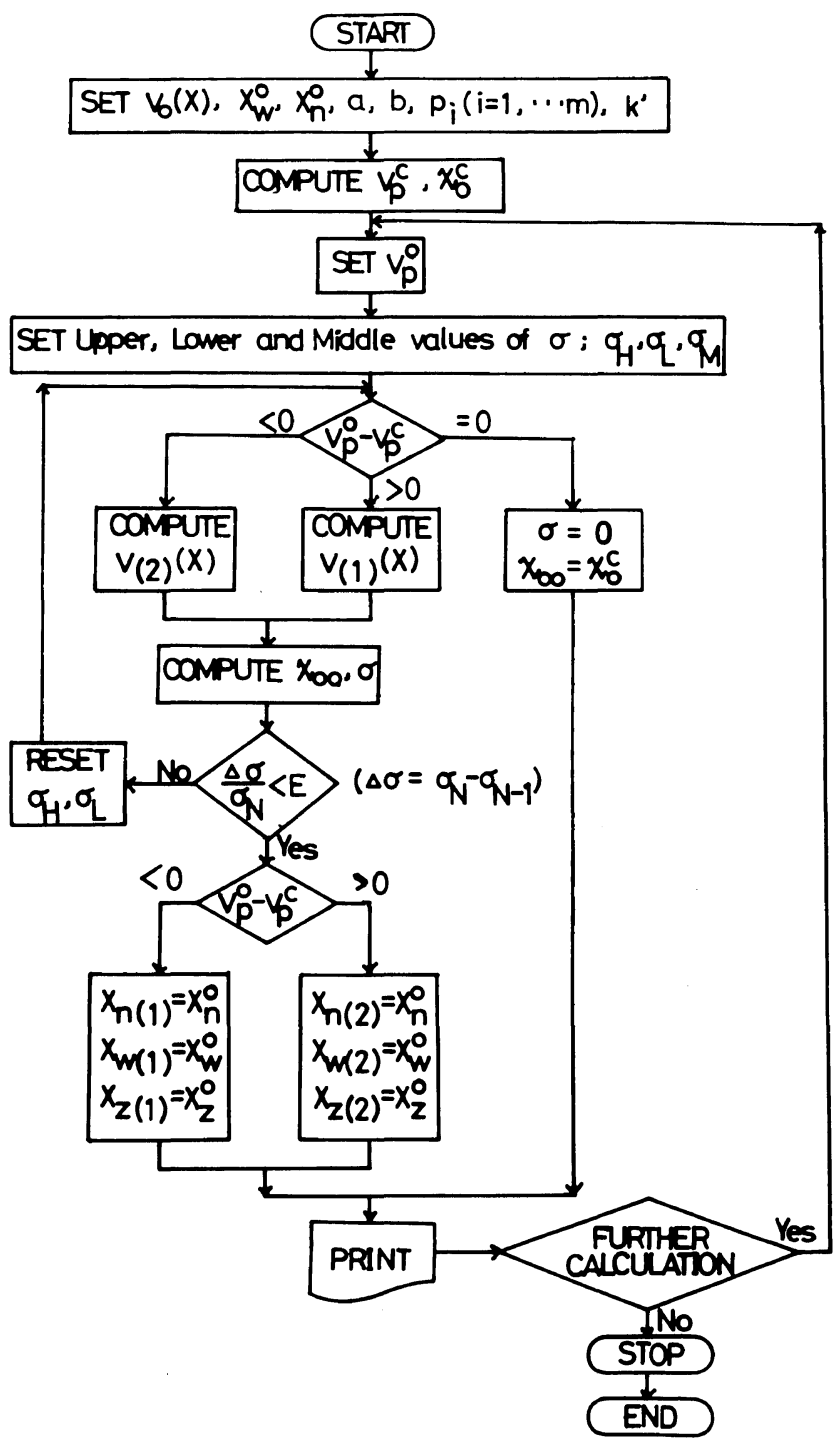

Figure 1. Flow chart for calculating the cloud point of a multicomponent polymer/single solvent system.

ments,

(2) Carry out computer simulation assuming arbitrarily chosen values of $p_{j}(j=1, \cdots, n)$ to obtain the relations between $\chi_{00}$ and $\rho_{\mathrm{p}}$,

(3) Construct the relations between $\chi_{00}$ and $1 / T$ using $\rho_{\mathrm{p}}$ vs. $T$ relations obtained in step (1) and $\chi_{00} v s . \rho_{\mathrm{p}}$ relations obtained in step (2) and determined $a$ and $b$ as the intercept and slope of $\chi_{00} v s$. $T$ plot, respectively,

(4) Calculate CPC ( $T_{\mathrm{cp}, \mathrm{c}}$ vs. $v_{\mathrm{p}}$ relations) using $a$ and $b$ obtained in step (3),

(5) Compute $\delta \equiv \sum_{N}\left(T_{\mathrm{cp}, \mathrm{c}}-T_{\mathrm{cp}, \mathrm{e}}\right)^{2} / N(N$ is the total number of the solutions, for which the cloud point was determined) and determine a set of $p_{j}(j=1, \cdots, n)$ to minimize $\delta$, (where $N \gg n$ ),

(6) Repeat steps $2-5$ and evaluate $a, b$, and $p_{j}$ where $\delta$ is below the permissible limit. 


\section{EXPERIMENTAL}

\section{Polymer}

The polystyrene (PS) sample of our study was Styron 666 (Asahi-Dow (now, Asahi Chemical Industry Co., Ltd.)) and also used in the previous papers. ${ }^{9,10,12,26}$ It was again characterized as follows: the weight-average molecular weight $M_{w}$ was $23.9 \times 10^{4}$ by gelpermeation chromatography (GPC) in tetrahydrofuran (THF) and $23.2 \times 10^{4}$ by light scattering in benzene at $25^{\circ} \mathrm{C}$; the numberaverage molecular weight $M_{n}$ was $8.6 \times 10^{4}$ by GPC in THF and $8.9 \times 10^{4}$ by membrane osmometry in toluene at $25^{\circ} \mathrm{C}$.

\section{Solvent}

Reagent grade cyclohexane $(\mathrm{CH})$ was dried over anhydrous calcium chloride before use.

\section{Cloud Point Determination}

Cloud point temperature $T_{\text {cp }}$ was determined visually (for dilute solutions) or with a $\mathrm{He}-\mathrm{Ne}$ laser beam (for concentrated solutions) or both (for an intermediate concentration range) within an accuracy of $\pm 0.03^{\circ} \mathrm{C}$ in a sealed capillary glass tube with inner diameter of $0.4 \mathrm{~cm}$ and filled nitrogen gas. The initial polymer volume fraction $v_{\mathrm{p}}^{0}$ was chosen from $6 \times 10^{-4}$ to 0.1509 . The detailed procedure is described elsewhere. ${ }^{27,28}$

\section{Determination of $\rho_{\mathrm{p}}$ vs. $T$ relation}

The relationships between $\rho_{\mathrm{p}}$ and the temperature $T$ were determined for three PS/CH systems with $v_{\mathrm{p}}^{0}=0.6,1.184$, and $1.737 \%$ to an accuracy of \pm 0.001 in $\rho_{\mathrm{p}}$ and $\pm 0.01^{\circ} \mathrm{C}$ in $T$. The detailed experimental procedure for evaluating $\rho_{\mathrm{p}}$ and have already been described. ${ }^{14}$

\section{RESULTS AND DISCUSSION}

Figure 2 shows the effect of $X_{w}^{0}$ on CPC, calculated from the solutions of four polymers (SZ, $X_{w}^{0} / X_{n}^{0}=1,1.1,2$, and 4) in a single solvent $\left(p_{1}=0.6, p_{2}=0\right.$, and $\left.k_{0}=0\right)$. The unfilled circle in the figure is the critical point and the filled circle, the threshold cloud point. The critical point, independently calculated by a

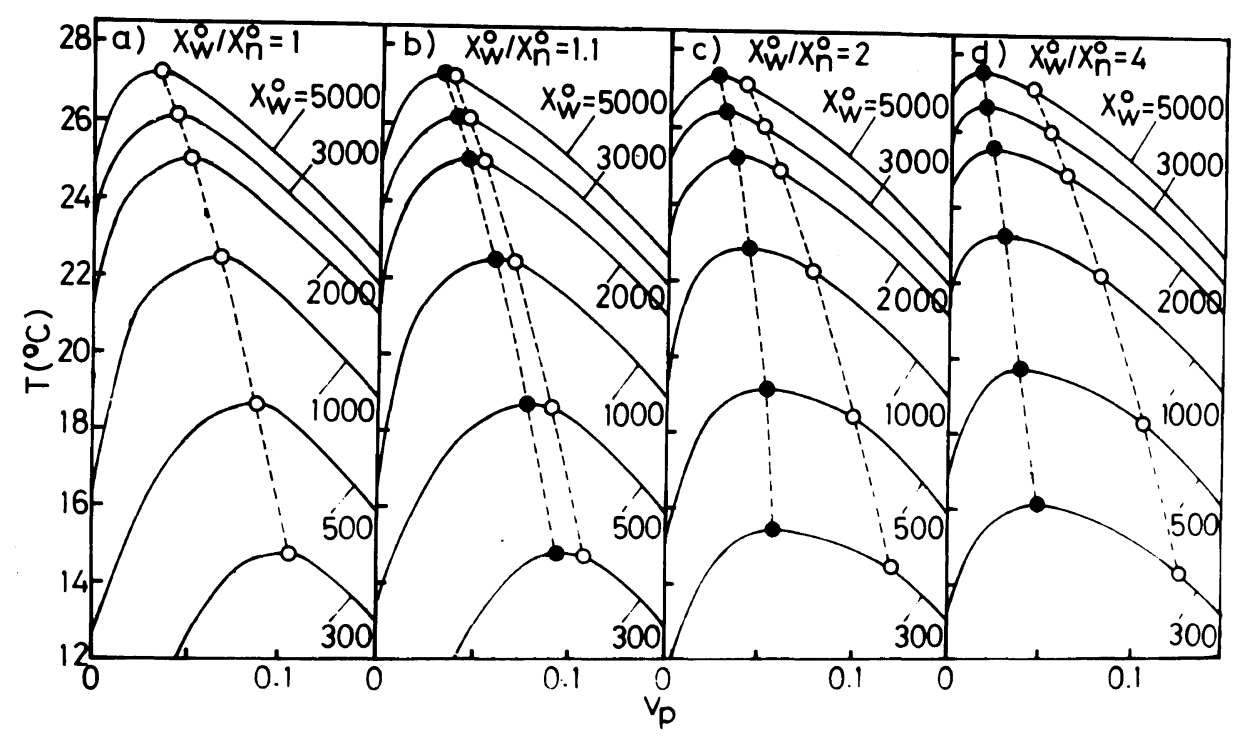

Figure 2. Effect of weight-average molar volume ratio $X_{w}^{0}$ of the polymer in a single solvent on cloud point curve $\left(p_{1}=0.5, p_{2}=0\right.$, and $\left.k_{0}=0\right): X_{w}^{0} / X_{n}^{0}=1$ (a), 1.1 (b), 2 (c), and 4 (d); $\bigcirc$, critical point; precipitation threshold point; number on curve denotes $X_{w}^{0}$. 
different procedure from CPC, lies just on the CPC. This verifies the reliability of the CPC calculation procedure used here.

A monodisperse polymer solution with low $X_{w}^{0}$ shows CPC having approximate symmetry, which disappears with increasing $X_{w}^{0}$ and $X_{w}^{0} / X_{n}^{0}$. As $X_{w}^{0}$ increases, CPC shifts to lower $v_{\mathrm{p}}$ and higher $T$ regions, showing a skewness in the former. The critical points $\left(T_{\mathrm{c}}\right.$ and $\bar{v}_{\mathrm{p}}^{\mathrm{c}}$ ) coincides very well as expected from the threshold points $\left(T_{\text {tcp }}\right.$ and $\left.v_{\mathrm{p}, \mathrm{tcp}}\right)$ for solutions of strictly monodisperse polymers. As the polydispersity of the polymer increases, the critical point moves to higher $v_{\mathrm{p}}$ region, but the threshold cloud point shifts to lower $v_{\mathrm{p}}$ region causing them to differ significantly. The deviation in the critical point from threshold cloud point increases remarkably with an increase in $X_{w}^{0} / X_{n}^{0}$ and slightly with a decrease in $X_{w}^{0}$. Note that this difference is significant even for a polymer with $X_{w}^{0} / X_{n}^{0}=1.1$, which is often regarded as "monodisperse polymer." Very careful attension should be given to this point in the case of a low $X_{w}^{0}$ polymer.

Figure 3 shows correlations between the difference, $\quad v_{\mathrm{p}, \mathrm{tcp}}-v_{\mathrm{p}}^{\mathrm{c}}\left(\equiv \Delta v_{\mathrm{p}}\right) \quad$ or $T_{\mathrm{tcp}}-T_{\mathrm{c}}$ $(\equiv \Delta T), X_{w}^{0}$ and $X_{w}^{0} / X_{n}^{0}$. The contour lines mean $\Delta T$ or $\Delta v_{\mathrm{p}}$. For polymers having sharp molecular weight distributions, both $\Delta v_{\mathrm{p}}$ and $\Delta T$ are predominantly controlled by the polymolecularity of the polymers. In contrast to this, $\Delta v_{\mathrm{p}}$ as well as $\Delta T$ are independent of $X_{w}^{0}$.

Figure 4 shows the effect of the polymolecularity of the original polymer dissolved is a single solvent on CPC. In the rather concentrated $v_{\mathrm{p}}$ region (i.e., $v_{\mathrm{p}}>v_{\mathrm{p}}^{\mathrm{c}}$ ), CPC in very insensitive to $X_{w}^{0} / X_{n}^{0}$ and in the range of $v_{p}$ smaller than $v_{\mathrm{p}}^{\mathrm{c}}$, it shifts to higher temperature possibly as a result to the precipitation of higher molecular weight components contained in polymers having large polymolecularity. Evidently, $T_{\text {tcp }}$ is much more sensitive than $T_{\mathrm{c}}$ to $X_{w}^{0} / X_{n}^{0}$. Effect of $X_{w}^{0} / X_{n}^{0}$ on CPC becomes less remarkable for larger $X_{w}^{0}$ polymer solutions.

Figure 5 shows the effects of $X_{w}^{0}$ and $X_{w}^{0} / X_{n}^{0}$

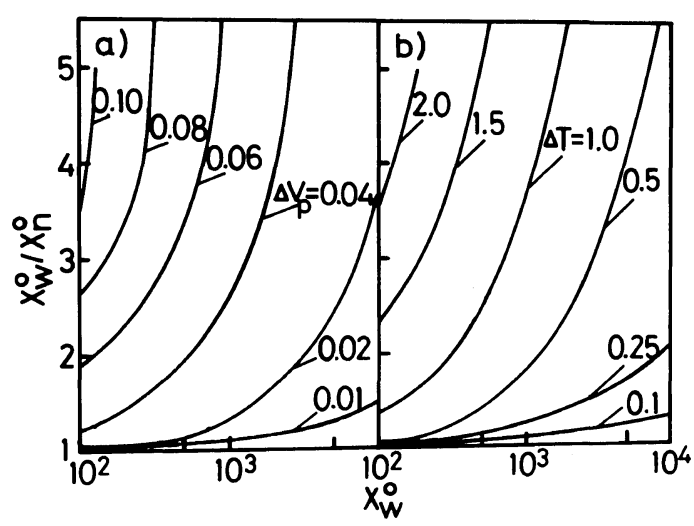

Figure 3. Relationships between the weight-average molar volume ratio $X_{w}^{0}$, ratio of $X_{w}^{0}$ to the numberaverage molar volume ratio $X_{n}^{0}\left(X_{w}^{0} / X_{n}^{0}\right)$ of the polymer and the difference in the polymer volume fraction $\Delta v_{\mathrm{p}}$ (a) in temperature $\Delta T$ (b) between the critical and precipitation threshold points.

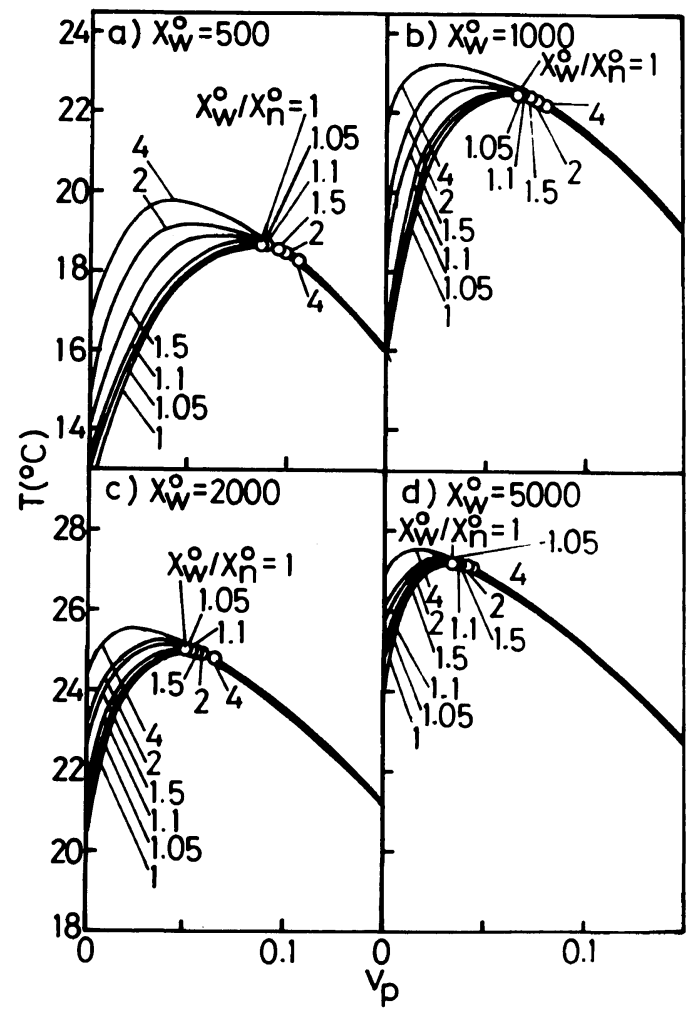

Figure 4. Effect of polydispersity expressed by $X_{w}^{0} / X_{n}^{0}$, of the polymer on the cloud point curves of polymers $\left(X_{w}^{0}=500(\mathrm{a}), 1000(\mathrm{~b}), 2000(\mathrm{c})\right.$, and $\left.5000(\mathrm{~d})\right)$ in a single solvent $\left(p_{1}=0.5, p_{2}=0\right.$, and $\left.k_{0}=0\right): \bigcirc$, critical point; number on each curve denotes $X_{w}^{0} / X_{n}^{0}$. 


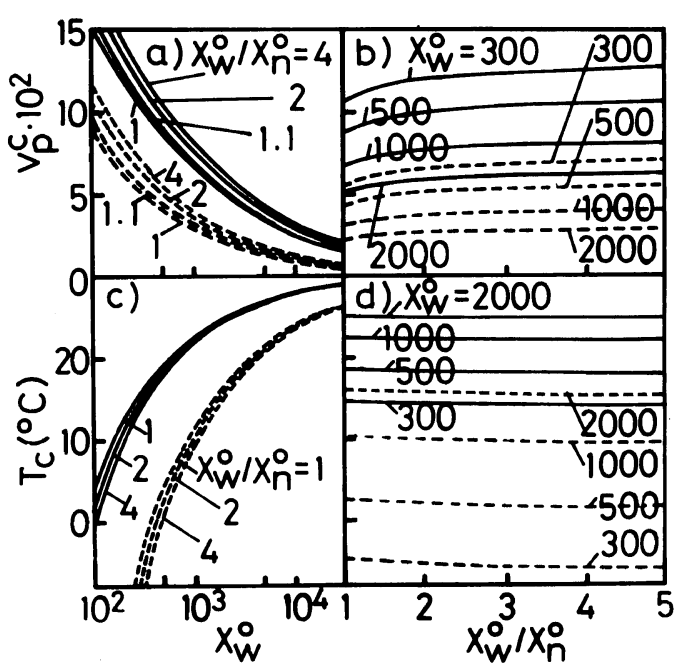

Figure 5. Effect of the weight-average molar volume ratio $X_{w}^{0}$ (a) and (c)) and the polymolecularity, $X_{w}^{0} / X_{n}^{0}$ (b) and d)) of the polymer on the critical concentration $v_{\mathrm{p}}^{\mathrm{c}}$ and temperature $T_{\mathrm{c}}$ of a polymer/single solvent system. ( $p_{1}=0$ (broken line) or 0.6 (full line), $p_{2}=0$, and $\left.k_{0}=0\right)$ : Number on the curve denote $X_{w}^{0} / X_{n}^{0}$ (a) and c)) or $X_{w}^{0}(\mathrm{~b})$ and d)).

on $v_{\mathrm{p}}^{\mathrm{c}}$ and $T_{\mathrm{c}}$. The broken line in the figure corresponds to $p_{1}=0$ and the full line, to $p_{1}=$ 0.6 . $v_{\mathrm{p}}^{\mathrm{c}}$ decreases monotonously and $T_{\mathrm{c}}$ increases sharply with $X_{w}^{0}$ irrespective of $X_{w}^{0} / X_{n}^{0}$. With an increase in $X_{w}^{0} / X_{n}^{0}, v_{\mathrm{p}}^{\mathrm{c}}$ increases gradually, especially for a polymer having small $X_{w}^{0}$ and small $X_{w}^{0} / X_{n}^{0}$, and $T_{\mathrm{c}}$ decreases very slowly. For example, for a polymer with $X_{w}^{0}=$ 300 , the variation in $v_{\mathrm{p}}^{\mathrm{c}}$ and $T_{\mathrm{c}}$ with change in $X_{w}^{0} / X_{n}^{0}$ from 1 to 5 was estimated to be $3.3 \%$ and $1.4^{\circ} \mathrm{C}$, respectively.

Figure 6 shows the effect of $p_{1}$ parameter on CPC, calculated for solutions of the polymers $\left(\mathrm{SZ}, X_{w}^{0}=300,500,1000\right.$ and $2000, X_{w}^{0} / X_{n}^{0}=1$ and 2$)$ in a single solvent $\left(p_{2}=p_{3}=\cdots=0\right.$ and $\left.k^{\prime}=0\right)$. The broken line is for a strictly monodisperse polymer and the full line, for $X_{w}^{0} / X_{n}^{0}=$ 2. CPC is remarkably influenced more by $p_{1}$ than the polymolecularity of the polymer, particularly in the case of a low $X_{w}^{0}$ polymer. An increase in $p_{1}$ brings about a rapid rise in the cloud point temperature, shifting $v_{\mathrm{p} \text {, tcp }}$ to a higher concentration range.

Figure 7 shows the effect of $p_{2}$ parameter on

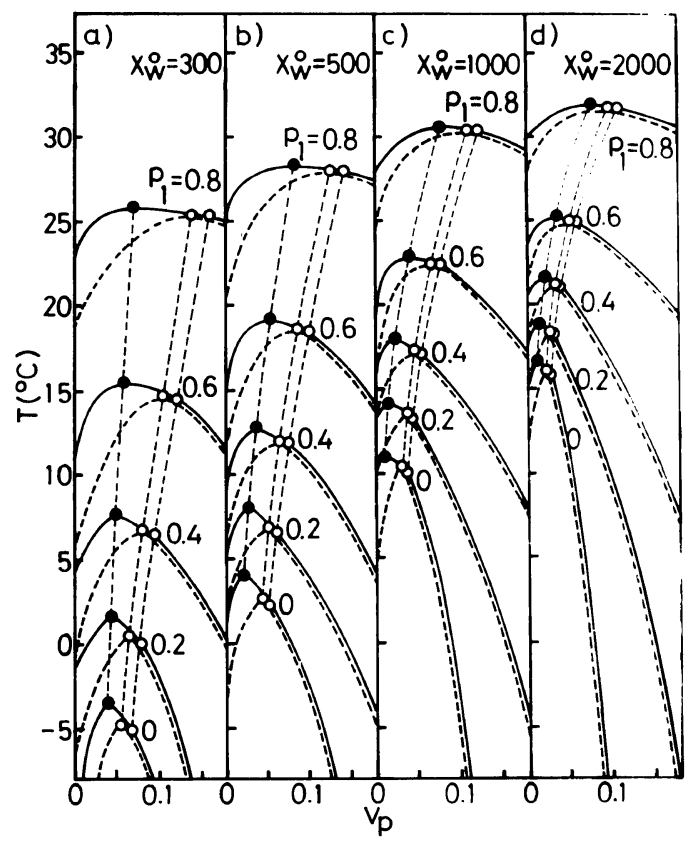

Figure 6. Effect of the concentration dependence parameter of the $\chi$-parameter on the cloud point curve of a polymer-single solvent system $\left(p_{1}=0\right.$ and $\left.k=0\right)$ : broken line, monodisperse polymer $\left(X_{m}^{0} / X_{n}^{0}=1\right)$; full line, $X_{w}^{0} / X_{n}^{0}=2 ; X_{w}^{0}=300$ (a), 500 (b), 100 (c), and 2000 (d); number on curve denotes $p_{1}$ : $\bigcirc$, critical point; $\bigcirc$, precipitation threshold point.

CPC, calculated for solutions of the polymers $\left(\mathrm{SZ}, X_{w}^{0}=300,500,1000\right.$, and $2000, X_{w}^{0} / X_{n}^{0}=1$ and 2$)$ in a single solvent $\left(p_{1}=0.6, p_{3}=p_{4}=\right.$ $\left.\cdots=0, k^{\prime}=0\right)$. The broken line corresponds to a strictly monodisperse polymer and the full line, to a polymer with $X_{w}^{0} / X_{n}^{0}=2 . p_{2}$ has less effect than $p_{1}$ on CPC.

Figure 8 shows the effect of the $p_{1}$ and $p_{2}$ parameters on the critical points. Both $v_{\mathrm{p}}^{\mathrm{c}}$ and $T_{\mathrm{c}}$ with $p_{1}$ and $p_{2}$, but the rate of increase in $v_{\mathrm{p}}^{\mathrm{c}}$ and $T_{\mathrm{c}}$ with $p_{1}$ or $p_{2}$ is small in the negative range of $p_{1}$ and $p_{2}$, both experimentally unrealistic for quasi-binary systems, but becomes extremely large for $p_{1}$ (or $\left.p_{2}\right) \gtrsim 0.5$. For an actual polymer system in a single solvent, $p_{1}$ is $0.6-0.7$ and hence, the effect of $p_{1}$ can never be ignored. Recently, Kamide and his coworkers $^{29-31}$ found that the characteristics of the phase equilibrium of a quasi-ternary sys- 


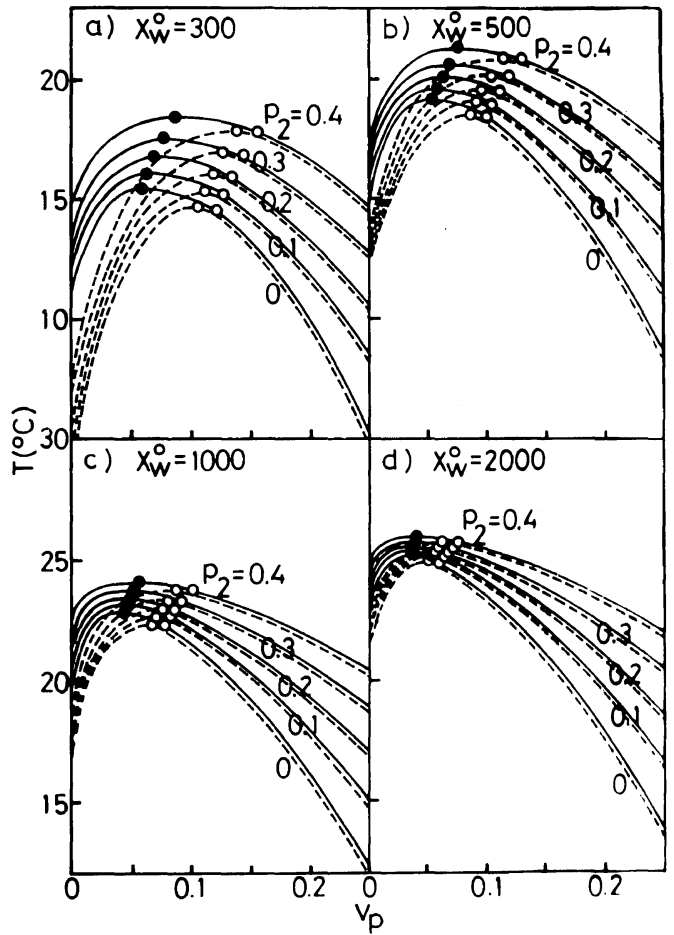

Figure 7. Effect of the concentration dependence parameter $p_{2}$ on the cloud point curve of the polymersolvent system ( $p_{1}=0.6$ and $\left.k=0\right)$; broken line, monodisperse polymer $\left(X_{w}^{0} / X_{n}^{0}=1\right)$; full line, $X_{w}^{0} / X_{n}^{0}=2 ; X_{w}^{0}=$ 300 (a), 500 (b), 1000 (c), and 2000 (d); number on curve denotes $p_{2} ; \bigcirc$, critical point; 9 , precipitation threshold point.

tem consisting of multicomponent polymers, solvent 1 and solvent 2 , can be reasonably approximated by those of a quasi-binary system having negative $p_{1}$. Figure 8 suggests strongly that, for a quasi-ternary system, the concentration dependence of the $\chi$-parameter plays only a minor role in the two-phase equilibrium, in contrast to the quasi-ternary system. The conclusions obtained for the case of $k^{\prime}=0$, as shown in Figures $2-8$ are expected to be valid even when $k^{\prime} \neq 0$, although the calculation of CPC with $k^{\prime} \neq 0$ is not always possible at present due to mathematical difficulty.

Figure 9 shows CPC calculated assuming various $p_{1}$ values and $p_{\mathrm{i}}(i \geq 2)=0$ for a $\mathrm{PS} / \mathrm{CH}$ system. In the figure, the unfilled circle is the

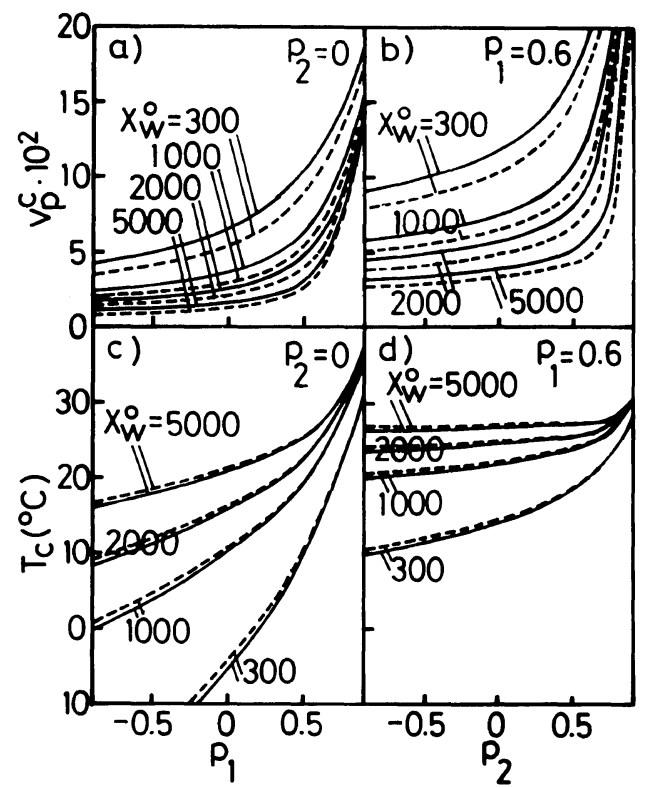

Figure 8. Dependence of the critical concentration $v_{\mathrm{p}}^{\mathrm{c}}$ and its temperature $T_{\mathrm{c}}$ of the polymer/single solvent system $(k=0)$ on $p_{1}$ and $p_{2}$ : broken line, $X_{w}^{0} / X_{n}^{0}=1$; full line $X_{w}^{0} / X_{n}^{0}=2 ; p_{2}=0$ (a) and c)) or $p_{1}=0.6(\mathrm{~b})$ and d)); number on curve denotes $X_{w}^{0}$.

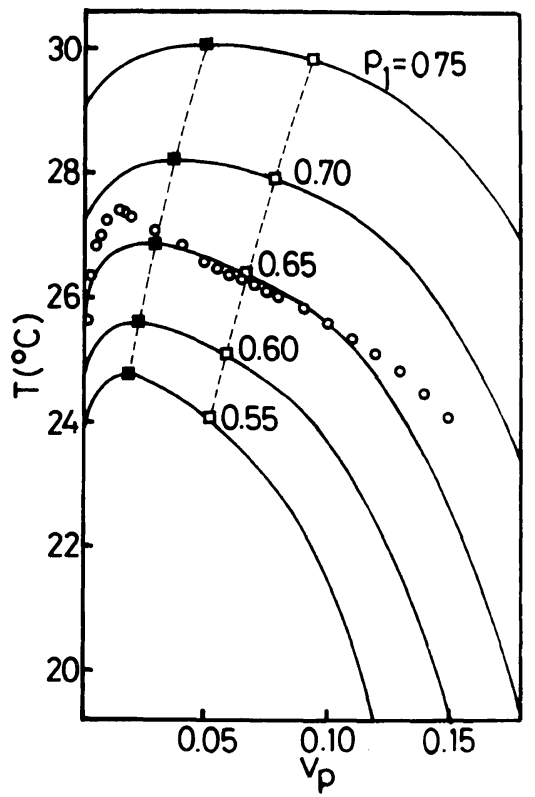

Figure 9. Cloud point curves of a polystyrene $\left(X_{w}=\right.$ 2117, $\left.X_{w}^{0} / X_{n}^{0}=2.8\right) /$ cyclohexane system: Full line, theoretical curve calculated using the value of $p_{1}$ shown on the curve and assuming $p_{2}=0$; circle, experimental data point. 


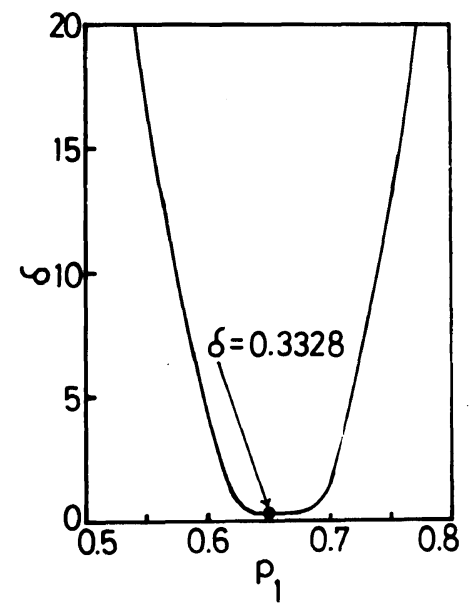

Figure 10. Plot of $\delta\left(\equiv \sum\left(T_{\mathrm{cp}, \mathrm{c}}-T_{\mathrm{cp}, \mathrm{e}}\right)^{2} / N\right)$ vs. $p_{1}$ for polystyrene/cyclohexane system: In this case, $p_{2}$ was assumed zero. Black point gives the optimum $p_{1}$ and its relative error $\delta\left(p_{1}=0.65, \delta=0.332\right)$.

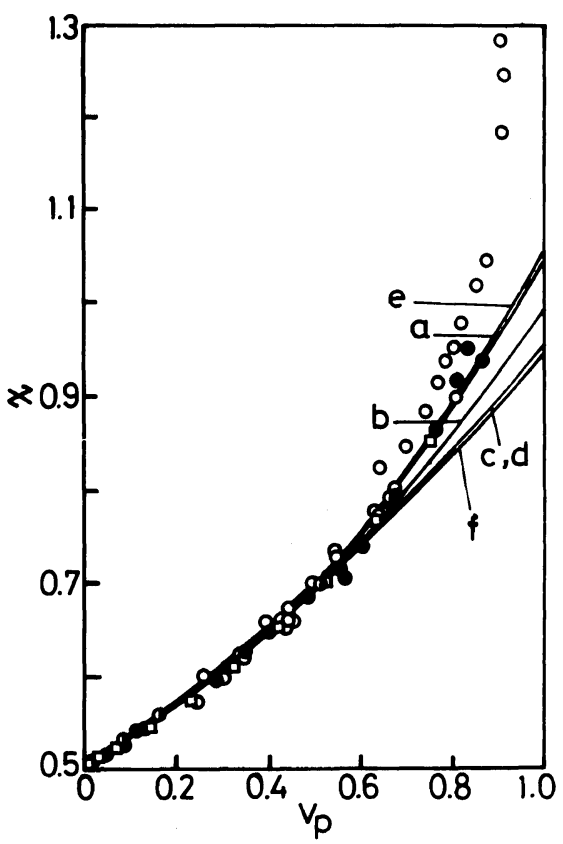

Figure 11. The concentration dependence of the $\chi$ parameter for a polystyrene/cyclohexane system: $O$, osmotic pressure by Krigboum-Geymer; $\mathbf{O}$, isothermal distillation by Krigbaum-Geymer; $\boldsymbol{O}$, vapor pressure by Krigbaum-Geymer; $\square$, ultracentrifuge by Scholte; lines a-f are calculated using $p_{1}$ and $p_{2}$, obtained by experiments; a, Krigbaum-Geymer; b, Scholte; c, Koningsveld et al. (1970); d, Koningsveld et al. (1971); e, Kuwahara et al.; f, this work.

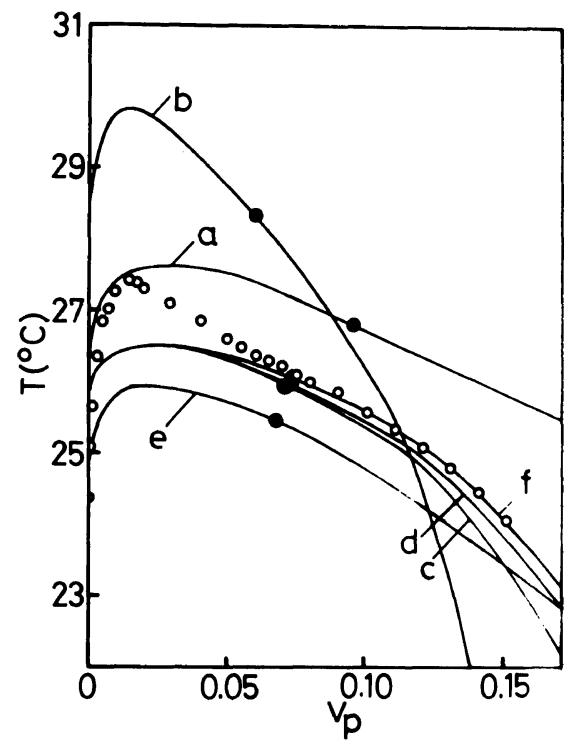

Figure 12. Cloud point curves of polystyrene/ cyclohexane system: Full line, theoretical curve calculated using the values of $a^{\prime}, b^{\prime}, p_{1}$ and $p_{2}$ summarized in Table I. Lines a- $f$ have the same meaning as those in Fig. 11. Unfilled circle, experimental data points.

experimental data point. Evidently, using a single $p_{1}$ we can never calculate CPC adequately for this system over a wide range of $v_{\mathrm{p}}$. In other words, $p_{1}$, which is suitable for representing the experimental CPC in a lower $v_{\mathrm{p}}$ region, overestimates $T_{\mathrm{cp}}$ in a higher $v_{\mathrm{p}}$ region and visa versa. $\delta$ was calculated from the figure and plotted against $p_{1}$ in Figure 10. $\delta$ attains a minimum $(0.333)$ at $p_{1}=0.65$, but, the agreement between the theoretical and experimental $\mathrm{CPC}$ is not satisfactory. This suggests strongly that, in contrast to the characteristics of the coexistence curve, CPC cannot be explained by a single $p_{1}$ alone.

The concentration dependence of $\chi$ parameter, evaluated for a $\mathrm{PS} / \mathrm{CH}$ system by many investigators, is plotted in Figure 11. In the figure, the curves have been calculated from the $p_{1}$ and $p_{2}$ values evaluated from the critical points. The experimental data points can be reasonably represented by eq 31 , in which terms higher than $v_{\mathrm{p}}{ }^{2}$ are neglected. That is, in the $v_{\mathrm{p}}$ range of $0-0.15$, both $p_{2}$ and 
Table I. Parameters $a^{\prime}, b^{\prime}, p_{1}$ and $p_{2}$ in eq 31 for a polystyrene-cyclohexane system $(T=299 \mathrm{~K})$

\begin{tabular}{lccccc}
\hline \multicolumn{1}{c}{ Author(s) } & $a^{\prime}$ & $b^{\prime}$ & $p_{1}$ & $p_{2}$ & References \\
\hline Krigbaum, Geymer (1959) & 0.2496 & 76.67 & $0.630_{4}$ & $0.480_{8}$ & 32 \\
Scholte (1970) & 0.2631 & 74.31 & $0.534_{4}$ & $0.430_{4}$ & 33 \\
Koningsveld et al. (1970) & 0.2035 & 90.50 & $0.610_{6}$ & $0.920_{7}$ & 34 \\
Koningsveld et al. (1971) & 0.2211 & 85.313 & $0.622_{2}$ & $0.289_{1}$ & 23 \\
Kuwahara et al. (1973) & 0.2798 & 67.50 & $0.607_{3}$ & $0.512_{1}$ & 28 \\
This work & $-0.0242_{4}$ & 158.79 & 0.643 & 0.200 & - \\
\hline
\end{tabular}

$p_{1}$ are necessary to represent the concentration dependence of $\chi$ and in a comparatively dilute range, there is no sharp distinction in $\chi$ between the investigators.

Figure 12 shows the $\mathrm{CPC}$ of a $\mathrm{PS} / \mathrm{CH}$ system. The unfilled circle is the actual experimental data and the full lines, theoretical curves calculated using the $p_{1}$ and $p_{2}$ parameters in the literature given in Table I. The curve calculated from $\chi$ value obtained by Krigbaum and Geymer ${ }^{32}$ can express experimental points in a lower $v_{\mathrm{p}}$ region, but overestimate them at a higher $v_{\mathrm{p}}$ region. The theoretical curve based on the $\chi$ of Scholte ${ }^{33}$ overestimates these points very much at $v_{\mathrm{p}}<0.12$ and under estimates them at $v_{\mathrm{p}}>0.12$. The total shape of CPC, calculated by $p_{1}$ and $p_{2}$ obtained by Koningsveld et al..$^{34}$ and Kuwahara et al. ${ }^{28}$ using the critical point data of Koningsveld et al.'s method, ${ }^{34}$ is similar to the actual experimental points, but their CPCs are underestimated throughout the entire range of $v_{\mathrm{p}}$ by $c a .1-1.5^{\circ} \mathrm{C}$. It is of interest that a very slight change in $p_{1}$ and $p_{2}$ cause remarkable variation in CPC. $p_{1}$ and $p_{2}$ should thus be estimated from CPC.

Figure 13 shows the relations between $\rho_{\mathrm{p}}$ and $T$, obtained by an actual phase separation experiment of $\mathrm{PS} / \mathrm{CH}$ system. The relations can be roughly approximated by a part of a circular arc with $\rho_{\mathrm{p}}$ approaching zero at specific temperature $T_{\mathrm{cp}}$ (i.e., the cloud point). As $v_{\mathrm{p}}$ increases, the $\rho_{\mathrm{p}} v s$. $T$ curve shifts to lower $T$ axis concurrently.

We carried out a computer experiment on

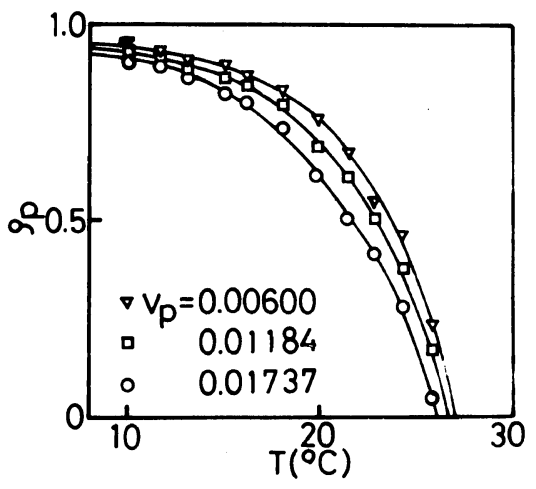

Figure 13. Experimental relations between $\rho_{\mathrm{p}}$ and $T$ for polystyrene $\left(X_{w}^{0}=2117, X_{w}^{0} / X_{n}^{0}=2.8\right)$ in cyclohexane. The polymer volume fractions of the starting solution $v_{\mathrm{p}}^{0}$ are $0.6 \times 10^{-2}(\nabla), 1.184 \times 10^{-2}(\square)$ and $1.737 \times 10^{-2}$ (O), respectively.

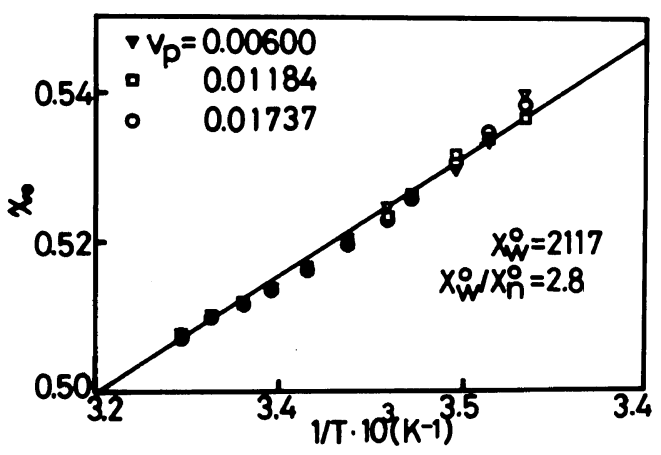

Figure 14. Temperature dependence of $\chi_{00}$ in eq 30 for polystyrene $\left(X_{w}^{0}=2117, X_{w}^{0} / X_{n}^{0}=2.8\right)$ in cyclohexane: $v_{\mathrm{p}}^{0} \times 10^{2}=0.60(\nabla), 1.184(\square)$ and $1.737(\bigcirc)$; full line, theoretical curve calculated by assuming $p_{1}=0.62, p_{2}=$ 0.20 and $k_{0}=0$.

two-phase equilibrium assuming variable $p_{1}$ and $p_{2}$ to obtain the relations between $\chi_{00}$ and $\rho_{\mathrm{p}}$, and converted them to those between $\chi_{00}$ 


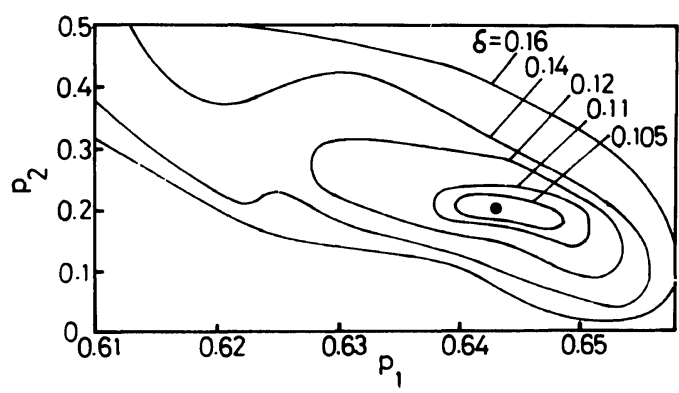

Figure 15. Relations among $p_{1}, p_{2}$ and $\delta$ for polystyrene/cyclohexane system: number denotes $\delta$ and the full line is the contour line of the same $\delta$.

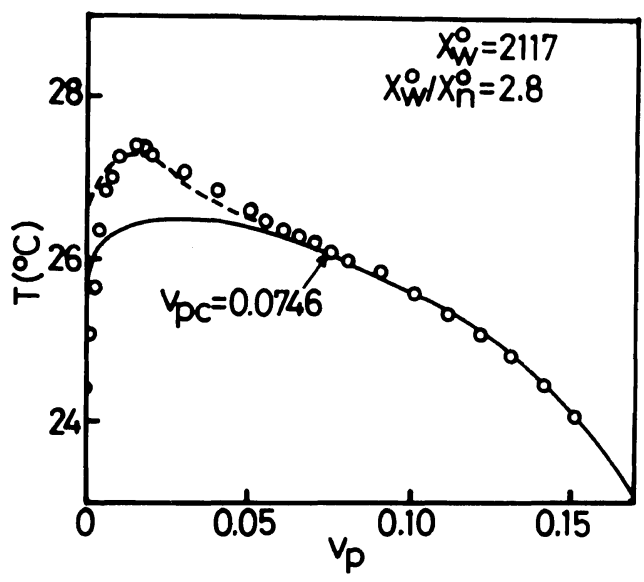

Figure 16. Cloud point curve of polystyrene $\left(X_{w}^{0}=\right.$ 2117, $X_{w}^{0} / X_{n}^{0}=2.8$ ) in cyclohexane: $\bigcirc$, experimental data; full line, theoretical curve calculated using $p_{1}=$ $0.643, p_{2}=0.200, k_{0}=0$; broken line, theoretical curve calculated using $p_{1}=0.643, p_{2}=0.200, \theta=307$ and $k_{0}=$ 108.

and $T$ by a comparison with the $\rho_{\mathrm{p}}-T$ relations in Figure 14, from which $a^{\prime}$ and $b^{\prime}$ were found for a given combination of $p_{1}$ and $p_{2}$.

Figure 15 shows the relations among $p_{1}, p_{2}$, and $\delta$, defined in the previous section, for the $\mathrm{PS} / \mathrm{CH}$ system. The most appropriate set of $p_{1}$ and $p_{2}$, giving the minimum $\delta(\simeq 0.1)$, could be determined as $p_{1}=0.643$ and $p_{2}=0.200$. These values are very near to the corresponding values evaluated by Kamide and Matsuda ${ }^{35}$ from the critical point data. The cloud point curve, calculated using these values of $a^{\prime}, b^{\prime}$, $p_{1}$, and $p_{2}$ can express accurately the experim- ental data except the threshold point region $\left(v_{\mathrm{p}} \simeq 0.07\right)$ as shown as full line in Figure 16. The maximum deviation of the theoretical $T_{\mathrm{c}}$ from the experiment is about $1^{\circ} \mathrm{C}$. In the figure the molecular weight dependence of $\chi$ parameter was not taken into account. The cloud point curve at lower $v_{\mathrm{p}}$ region is expected to be significantly infulenced by the large molecular weight components and the above inconsistence at lower $v_{\mathrm{p}}$ region may be due to the neglect of the molecular weight dependence on $\chi$-parameter.

Kamide et al. ${ }^{12}$ evaluated for $\mathrm{PS} / \mathrm{CH}$ system $k_{0}=108$ and $\theta=307 \mathrm{~K}$. We calculated the cloud point curve using these values together with $\alpha, \beta, p_{1}$, and $p_{2}$ values determined before and shown as broken line in Figure 16. Inspection of Figure 16 leads us to the conclusion that the experimental cloud point data of the polydisperse polymer in a single solvent can be reasonably represented by the theory, in which the polydispersity of the polymer and the concentration- and the molecular weightdependence of the $\chi$-parameter are taken into consideration.

\section{APPENDIX A}

\section{Derivation of Eq 5}

The chemical potentials of the solvent and $X$-mer, $\Delta \mu_{0}$ and $\Delta \mu_{i}(i=1, \cdots, m)$ for a multicomponent polymer-single solvent system are given $b^{36}$

$$
\Delta \mu_{0}=R T\left\{\ln \left(1-v_{\mathrm{p}}\right)+\left(1-\frac{1}{X_{n}}\right) v_{\mathrm{p}}+\chi v_{\mathrm{p}}^{2}\right\}
$$

and

$$
\begin{aligned}
\Delta \mu_{i}= & R T\left\{\ln v_{i}+\left(X_{i}-1\right)+X_{i}\left(1-\frac{1}{X_{n}}\right) v_{\mathbf{p}}\right\} \\
& +\frac{\partial \Delta H}{\partial N_{i}}
\end{aligned}
$$

where $N_{i}$ is the number of $X_{i}$-mer moles. Substituting eq 2 into eq A-1, we obtain eq 4 . 
In eq $\mathrm{A}-1, \chi v_{\mathrm{p}}{ }^{2}$ must be of the form

$$
R T \chi v_{\mathrm{p}}^{2}=\frac{\partial \Delta H}{\partial N_{0}}
$$

where $N_{0}$ is the number of solvent moles. The necessary and sufficient 'condition' under which $\Delta \mu_{0}$ and $\Delta \mu_{i}$ satisfy the Gibbs-Duhem relation

$$
N_{0} \mathrm{~d}\left(\Delta \mu_{0}\right)+\sum_{i=1}^{m} N_{i} \mathrm{~d}\left(\Delta \mu_{i}\right)=0
$$

is

$$
\frac{\partial \Delta H}{\partial N_{i}}=\frac{\partial}{\partial N_{i}}\left\{\int_{0}^{N_{0}}\left(\frac{\partial \Delta H}{\partial N_{0}}\right) \mathrm{d} N_{0}\right\}
$$

On substituting eq 2 into eq A-5 we obtain

$$
\begin{aligned}
\frac{1}{R T} \cdot \frac{\partial \Delta H}{\partial N_{i}}= & \chi_{00} X_{i}\left(1+\frac{k^{\prime}}{X_{n}}\right)\left\{\left(1-v_{\mathrm{p}}\right)^{2}\right. \\
& \left.+\sum_{j=1}^{m} p_{j}\left(\frac{1}{j+1}-\frac{j+2}{j+1} v_{\mathrm{p}}^{j+1}+v_{\mathrm{p}}^{j+2}\right)\right\} \\
& +\chi_{00} k^{\prime} X_{i}\left(\frac{1}{X_{i}}-\frac{1}{X_{n}}\right)\left\{\left(1-v_{\mathrm{p}}\right)\right. \\
& \left.+\sum_{j=1}^{m} \frac{p_{j}}{j+1}\left(1-v_{\mathrm{p}}^{j+1}\right)\right\}
\end{aligned}
$$

Combinating eq A-6 with eq A-2 leads to eq 5.

\section{APPENDIX B}

Deviation of $E q 27$ and 28

Substitution of eq 26 into 25 leads to

$$
\begin{aligned}
& \frac{V_{0}}{R T} \overline{\Delta G_{i j}^{\prime}}= \frac{1}{1-v_{\mathrm{p}}}-\chi_{00}\left\{1+k^{\prime}\left(1+\frac{1}{X_{n}}-\frac{X_{w}}{X_{n}}\right)\right\} \\
&\left\{2+\sum_{q=1}^{n} p_{\mathrm{q}}(q+2) v_{\mathrm{p}}^{q}\right\} \equiv M \\
& \text { for } i \neq j \quad(\mathrm{~B}-1)
\end{aligned}
$$

or

$$
\frac{V_{0}}{R T} \overline{\Delta G_{i i}^{\prime}}=M+\frac{1}{X_{i} v_{i}} \equiv M+M_{i} \quad \text { for } \quad i=j
$$

From eq B-1, B-2, and eq 22, we obtain

$$
\begin{aligned}
\left|\Delta G^{\prime}\right| & =\left(\frac{R T}{V_{0}}\right)^{m} \\
& \left|\begin{array}{cccc}
M+M_{1} & M & \ldots & M \\
M & M+M_{2} & \cdots & M \\
\vdots & \vdots & \vdots \\
M & M & M+M_{m}
\end{array}\right| \\
& =\left(\frac{R T}{V_{0}}\right)^{m}\left(\prod_{i=1}^{m} M_{i}\right)\left(1+M \sum_{i=1}^{m} \frac{1}{M_{i}}\right)=0
\end{aligned}
$$

Then, we have

$$
1+M \sum_{i=1}^{m} \frac{1}{M_{i}}=0
$$

for the spinodal. Rearrangement of eq B-4 with the aid of eq B-1 and B-2 gives eq 27 . In deriving eq B-3, we employed the relation

$$
|D|=\left|\begin{array}{cccc}
x_{1} & a & \cdots & a \\
a & x_{2} & \cdots & a \\
\vdots & \vdots & & \vdots \\
a & a & \cdots & x_{m}
\end{array}\right|=f(a)-\left.a \frac{\mathrm{d} f(X)}{\mathrm{d} X}\right|_{x=a}
$$

where

$$
f(x)=\prod_{i=1}^{m}\left(x_{i}-x\right)
$$

Differentiating eq B-3 by $v_{i}$, we obtain

$\frac{\partial\left|\Delta G^{\prime}\right|}{\partial v_{i}}$

$$
=\left(\frac{R T}{V_{0}}\right)^{m}\left(\prod_{i=1}^{m} M_{i}\right)\left(\frac{\partial M}{\partial v_{\mathrm{p}}} v_{\mathrm{p}} X_{w}-\frac{X_{i}}{X_{w} v_{\mathrm{p}}}\right) \equiv W_{i}
$$

Then, from eq B-1, B-2, B-7, and 23 and using eq B-5 and B-6 we obtain

$$
\left|\Delta G^{\prime \prime}\right|=(-1)^{2} W_{1}
$$

$$
\left|\begin{array}{cccc}
M+M_{2} & M & \cdots & M \\
M & M+M_{3} & \cdots & M \\
\vdots & \vdots & & \\
M & M & \cdots & M+M_{m}
\end{array}\right|
$$




$$
\begin{gathered}
+\sum_{j=2}^{m} W_{j}(-1)^{j+1}(-1)^{j}\left|\begin{array}{ccccc}
M & M & & & \\
M & M+M_{2} & & & \\
\vdots & \vdots & \ddots & & \\
M & M & M+M_{j-1} & M & \\
M & M & M & M+M_{j+1} & \\
\vdots & \vdots & & \ldots & \\
M & M & & M+M_{m}
\end{array}\right| \\
=-\left(\prod_{i=1}^{m} M_{i}\right)\left(\frac{M}{M_{i}}\right)\left(\sum_{i=1}^{m} \frac{W_{i}}{M_{i}}\right)=0
\end{gathered}
$$

Equation B-8 can be rewritten, using eq B-1 and $\mathrm{B}-2$, as eq 28 .

Acknowledgement. The authors should like to express their sincere gratitude to Dr. Yukio Miyazaki of Textile Research Laboratory, Asahi Chemical Industry Co., Ltd. and to Dr. Ken Sugamiya of Asahikawa University for their helpful comments.

\section{REFERENCES}

1. J. N. Bronsted and K. Volquartz, Trans. Faraday Soc., 35, 576 (1939).

2. R. B. Richards, Trans. Faraday Soc., 42, 10, 20 (1946).

3. A. R. Shultz and P. J. Flory, J. Am. Chem. Soc., 74, 4760 (1952).

4. K. Kamide and K. Sugamiya, Makromol. Chem., 139, 197 (1970).

5. K. Kamide and K. Sugamiya, Makromol. Chem., 156, 259 (1972).

6. K. Kamide, Y. Miyazaki, and K. Yamaguchi, Makromol. Chem., 173, 157 (1973).

7. K. Kamide, Y. Miyazaki, and K. Yamaguchi, Makromol. Chem., 173, 175 (1973).

8. K. Kamide and Y. Miyazaki, Makromol. Chem., 176, 1447 (1975).

9. K. Kamide, Y. Miyazaki and T. Abe, Makromol: Chem., 177, 485 (1976).

10. K. Kamide, Y. Miyazaki, and T. Abe, Polym. J., 9, 395 (1977).

11. K. Kamide and Y. Miyazaki, Polym. J., 13, 325 (1981).

12. K. Kamide, T. Abe, and Y. Miyazaki, Polym. J., 14, 355 (1982).

13. G. Rehage, D. Moller, and O. Ernst, Makromol. Chem., 38, 232 (1965).
14. N. Kuwahara, M. Nakata, and M. Kaneko, Polymer, 14, 415 (1973).

15. R. Koningsveld and A. J. Staverman, Kolloid-Z. Z. Polym., 218, 114 (1967).

16. R. Koningsveld and A. J. Staverman, J. Polym. Sci., $A-2,6,305$ (1968).

17. R. Koningsveld and A. J. Staverman, J. Polym. Sci. A-2, 6, 349 (1968).

18. M. Gordon, H. A. G. Chermin, and R. Koningsveld, Macromolecules, 2, 107 (1969).

19. R. Koningsveld, W. H. Stockmayer, J. W. Kennedy, and L. A. Kleintjens, Macromolecules, 7, 73 (1974).

20. K. Kamide, T. Ogawa, and M. Matsumoto, Kobunshi Kagaku, 25, 788 (1968).

21. K. Kamide, T. Ogawa, M. Sanada, and M. Matsumoto, Kobunshi Kagaku, 25, 440 (1968).

22. R. Koningsveld and A. J. Staverman, J. Polym. Sci., $A-2,6,325$ (1968).

23. R. Koningsveld and L. A. Kleintjens, Macromolecules, 4, 637 (1971).

24. See, for example, M. Kurata, "Thermodynamics of Polymer Solution," Harwood Academic Pubs., Chur, London, New York, 1982, Chapter 2.

25. W. H. Stockmayer, J. Chem. Phys., 17, 588 (1949).

26. K. Kamide, K. Sugamiya, T. Ogawa, C. Nakayama, and N. Baba, Makromol. Chem., 135, 23 (1970).

27. T. Dobashi, M. Nakata, and M. Kaneko, J. Chem. Phys., 72, 6685 (1970).

28. S. Saeki, N. Kuwahara, S. Konno, and M. Kaneko, Macromolecules, 6, 246 (1973).

29. K. Kamide, S. Matsuda, and Y. Miyazaki, Polym. J., 16, 479 (1984).

30. K. Kamide and S. Matsuda, Polym. J., 16, 515 (1984).

31. K. Kamide and S. Matsuda, Polym. J., 16, 591 (1984).

32. W. R. Krigbaum and D. Q. Geymer, J. Am. Chem. Soc., 81, 1859 (1959).

33. T. G. Scholte, J. Polym. Sci., A-2, 8, 841 (1970).

34. R. Koningsveld, L. A. Kleintjens, and A. R. Shultz, 


\section{CPC and CP of Multicomponent Polymer/Solvent System}

J. Polym. Sci., A-2, 8, 1261 (1970).

35. K. Kamide and S. Matsuda, Polym. J., 16, 807 (1984).
36. See, for example, P. J. Flory, "Principle of Polymer Chemistry," Cornell Univ. Press, Ithaca, N.Y., 1953. 